\title{
Cost analysis of mydriasis strategies in cataract surgery care in the Netherlands
}

Citation for published version (APA):

Simons, R. W. P., Rondas, L. U. E., van den Biggelaar, F. J. H. M., Berendschot, T. T. J. M., Visser, N., de Crom, R. M. P. C., \& Nuijts, R. M. M. A. (2021). Cost analysis of mydriasis strategies in cataract surgery care in the Netherlands. Journal of Cataract and Refractive Surgery, 47(8), 982-990. https://doi.org/10.1097/j.jcrs.0000000000000577

Document status and date:

Published: 01/08/2021

DOI:

10.1097/j.jcrs.0000000000000577

Document Version:

Publisher's PDF, also known as Version of record

Document license:

Taverne

Please check the document version of this publication:

- A submitted manuscript is the version of the article upon submission and before peer-review. There can be important differences between the submitted version and the official published version of record.

People interested in the research are advised to contact the author for the final version of the publication, or visit the DOI to the publisher's website.

- The final author version and the galley proof are versions of the publication after peer review.

- The final published version features the final layout of the paper including the volume, issue and page numbers.

Link to publication

\footnotetext{
General rights rights.

- You may freely distribute the URL identifying the publication in the public portal. please follow below link for the End User Agreement:

www.umlib.nl/taverne-license

Take down policy

If you believe that this document breaches copyright please contact us at:

repository@maastrichtuniversity.nl

providing details and we will investigate your claim.
}

Copyright and moral rights for the publications made accessible in the public portal are retained by the authors and/or other copyright owners and it is a condition of accessing publications that users recognise and abide by the legal requirements associated with these

- Users may download and print one copy of any publication from the public portal for the purpose of private study or research.

- You may not further distribute the material or use it for any profit-making activity or commercial gain

If the publication is distributed under the terms of Article $25 \mathrm{fa}$ of the Dutch Copyright Act, indicated by the "Taverne" license above, 


\title{
Cost analysis of mydriasis strategies in cataract surgery care in the Netherlands
}

\author{
Rob W.P. Simons, MD, Luigi U.E. Rondas, MD, Frank J.H.M. van den Biggelaar, PhD, Tos T.J.M. Berendschot, PhD, \\ Nienke Visser, $M D$, PhD, Ronald M.P.C. de Crom, MD, Rudy M.M.A. Nuijts, MD, PhD
}

Purpose: To investigate the economic impact of an intracameral mydriatics and anesthetic agent (ICMA), topical mydriatics, and a mydriatic ocular insert in cataract patients.

Setting: One public hospital in the Netherlands.

Design: Prospective cohort study.

Methods: Resource use data were collected from a healthcare and societal perspective on the day of surgery. Other outcome parameters included pupil size, surgeon satisfaction, postoperative pain, and Catquest-9SF scores.

Results: A total of 368 patients were included, the mean costs per patient were $€ 506$ in the ICMA group ( $n=122$ ), $€ 474$ in the ocular insert group ( $n=115$ ), and $€ 451$ in the topical group ( $n=131)$. The acquisition cost of ICMA was highest and resulted in longer surgical time. After correction for an imbalance in the distribution of fast and slow surgeons, the mean costs in the ocular insert and topical groups were comparable (€450 vs $€ 444)$. There was no statistically significant difference in the use of additional mydriatics intraoperatively ( $P=.521)$. The mean ratio of pupil size to white-to-white distance was lower in the ICMA group during all intraoperative measurements $(P<.001)$ but similar between the topical and ocular insert groups ( $P$ range .11-.82).

Conclusions: In the investigated setting in the Netherlands, ICMA was the most costly strategy. In addition, pupil size was lowest in the ICMA group but did not result in more additional mydriasis measures intraoperatively. The ocular insert was comparable with topical mydriatics in costs and pupil size. Implementation of ICMA could be considered when availability of nurses or physical space for perioperative care is limited.

J Cataract Refract Surg 2021; 47:982-990 Copyright ( 2021 Published by Wolters Kluwer on behalf of ASCRS and ESCRS

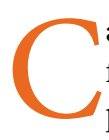
ataract surgery is one of the most frequently performed types of surgery with more than 180000 procedures annually in the Netherlands. ${ }^{1}$ In 2017, the costs of cataract care were $€ 317$ million in the Netherlands, of which $76 \%$ was hospital bound. ${ }^{2}$ Clearly, small efficiency gains in cataract care at the patient level may lead to substantial cost savings on a macrolevel.

One potential area for improvement in cataract care is the perioperative process. Sufficient and stable mydriasis is crucial to perform safe and efficient cataract surgery. Traditionally, mydriasis has been achieved preoperatively using topical mydriatics, such as phenylephrine and tropicamide, in various protocols that differ regarding timing and frequency. However, this requires patients to arrive at the surgical center well in advance of the surgery and trained personnel to administer the mydriatics. Furthermore, topical mydriatics may interfere with ocular surface integrity and intraoperative visualization and pose a risk for systemic side effects, such as tachycardia and hypertension. ${ }^{3}$

Alternative methods to achieve mydriasis have been introduced to the market. The mydriatic ocular insert (Mydriasert) is a small insert that is applied to the lower conjunctival sac preoperatively for 30 to 45 minutes, slowly releasing $5.4 \mathrm{mg}$ of phenylephrine hydrochloride and $0.28 \mathrm{mg}$ of tropicamide. Although it still requires patients to arrive early at the surgical center, it reduces the work load for nurses. Recently, an intracameral mydriatics and anesthetic agent (ICMA; Mydrane) was approved for the European market for use in adult patients undergoing cataract surgery. It contains tropicamide $0.02 \%$, phenylephrine hydrochloride $0.31 \%$, and lidocaine hydrochloride $1 \%$ and is injected intracamerally by the surgeon immediately after the first incision to induce rapid mydriasis and provide additional anesthesia. The efficacy and safety of

Funded by Laboratoires The'a (Clermont-Ferrand, France). The funding organization reviewed the study protocol and manuscript but was not involved in study conduct; collection, management, analysis, and interpretation of the data; preparation of the manuscript; and decision to submit the manuscript for publication.

Corresponding author: Rob W.P. Simons, MD, University Eye Clinic Maastricht, Maastricht University Medical Center, P.O. Box 5800, 6202 AZ Maastricht, the Netherlands. Email: rob.simons@mumc.nl. 
ICMA and the ocular insert have been investigated in a number of studies and were found to be similar to topical mydriatics. $^{4,5}$

Although the acquisition cost of ICMA is higher than the cost of topical mydriatics and the ocular insert, its use reduces the workload for nurses, which might potentially reduce costs. It also obviates the need for patients to arrive early at the surgical center. A previous budget impact model in the U.K. healthcare setting showed that use of ICMA in cataract care is cost saving. ${ }^{6}$ In addition, a recent study showed that implementation of ICMA resulted in shorter surgical times and shorter rotation times between patients. ${ }^{7}$ The primary aim of this study was to investigate the economic impact of ICMA compared with topical mydriatics and the ocular insert in patients undergoing cataract surgery at 1 public hospital in the Netherlands.

\section{METHODS}

This study was conducted between February and September 2019 in 1 public hospital in the Netherlands (Zuyderland Medical Center, Heerlen). The institutional review board of the surgical center ruled out that approval was not required. The study was performed in accordance with the tenets of the Declaration of Helsinki. All participants gave written informed consent after the nature of the study had been fully explained.

\section{Study Design}

The study was conducted as a nonrandomized comparative study. Consecutive patients undergoing cataract surgery were included in the study. The first cohort of patients included in the study was allocated to the topical mydriatics group, which was the standard mydriatic strategy at the surgical center. After a predetermined number of patients were included, a second cohort of patients was allocated to the ocular insert group, followed by a third patient cohort to ICMA. Inclusion commenced immediately after switching to a new mydriatic agent, without a period to account for potential learning curves. During the study, no changes were made in perioperative processes.

\section{Inclusion and Exclusion Criteria}

Patients undergoing cataract surgery under topical anesthesia were eligible for inclusion. Exclusion criteria included the following: inability to comply with study procedures or complete follow-up; cataract surgery combined with another ophthalmic surgical procedure; ophthalmic disorders (other than senile cataract) that might affect visual acuity or interfere with surgery; known incomplete mydriasis $(<5.5 \mathrm{~mm})$ and/or patients requiring mechanical mydriasis (tested preoperatively using topical mydriatics); and any contraindications to the anesthetic and/or mydriatic agents used in the study.

\section{Study Procedures}

All patients were requested to arrive at the waiting area of the surgical center well in advance of the surgery, regardless of the mydriatic used. Patients allocated to the topical mydriatics group were administered tropicamide $0.5 \%$ (Bausch \& Lomb, Inc.), phenylephrine hydrochloride 10\% (Bausch \& Lomb, Inc.), and cyclopentolate hydrochloride $1 \%$ (Bausch \& Lomb, Inc.) 3 times every 10 minutes by a trained nurse. In patients allocated to the ocular insert, the insert was placed into the inferior conjunctival sac by a trained nurse. The insert was removed by the nurse when the patient was transported to the operating room. Patients allocated to the ICMA group received a single $200 \mu \mathrm{L}$ of the agent into the anterior chamber immediately after the first incision was made by the surgeon. The surgeon proceeded with capsulorhexis when mydriasis was deemed sufficient.

All surgeries were performed under topical anesthesia by a total of 10 senior cataract surgeons (at least 300 cataract surgeries annually) using a standard phacoemulsification technique with subsequent intraocular lens implantation. If mydriasis was inadequate, surgeons were allowed to use additional methods for mydriasis at their own discretion, for example, topical mydriatics, intracameral phenylephrine hydrochloride, or pupillary expansion device (Malyugin ring or iris hooks).

\section{Outcome Assessment}

Cost Analysis The primary outcome was incremental costs. The cost analysis was performed according to national guidelines for economic evaluations. ${ }^{8}$ Data on resource use were collected from a healthcare and societal perspective within a time horizon of 1 day (the day of surgery). Costs were determined by multiplying volumes of resource use with unit cost prices including sales taxes. All costs were converted to a common price level (2019 euros $[€])$ using the Dutch consumer price index. ${ }^{9}$ The following types of hospital resource use were included in the cost analysis: mydriatic agent, additional materials to administer ICMA (syringe and hydrodissection needle), operating room time, additional intraoperative mydriatics, and nurse time spent on administration of mydriatics and postoperative care relating to pain. Time spent for waiting in the preoperative waiting area was not accounted for in the cost analysis. A cost questionnaire was used to determine out-of-hospital resource use on the day of surgery and included unplanned outpatient visits and calls, travel costs, postoperative analgesics, and informal care.

Costs of medication, including mydriatics, were valued using reimbursement prices. ${ }^{10}$ Costs of additional materials used to administer ICMA were based on actual purchase prices paid by the hospital (hydrodissection needle) or wholesale prices (syringe). ${ }^{11}$ Operating room time was valued using integral cost prices provided by the hospital and included personnel, materials, and overhead costs. Two costs drivers were applicable: general operating room costs (cost per minute spent in the operating room) and ophthalmology costs (cost per minute spent in surgery). The cost of pupillary expansion devices (Malyugin ring) was based on the actual purchase price paid by the hospital. Nurse time, outpatient visits and calls, travel costs, and informal care were valued using standardized prices recommended by the Dutch guidelines for cost analyses. ${ }^{8}$

Pupil Diameter All surgeries were recorded on video and subsequently assessed by a single examiner. Video stills were made prior to 5 intraoperative stages: just before the first corneal incision (T1), just before injection of the ophthalmic viscosurgical device (T2), just before capsulorhexis (T3), just before intraocular lens implantation (T4), and just before the end of the surgery (injection of cefuroxime; T5). ${ }^{5}$ The video stills were imported into image editing software, and the ruler function was used to measure the horizontal white-to-white distance and the horizontal pupil diameter in number of pixels. 
Subsequently, the ratio of pupil size to white-to-white distance was calculated. This ratio was also converted to millimeters assuming a mean white-to-white distance of $11.9 \mathrm{~mm}$. This enabled determination of the proportion of patients with a pupil diameter of $6 \mathrm{~mm}$ or more, which is considered sufficient to safely perform capsulorhexis. ${ }^{5}$

Surgeon-Reported Outcomes Surgeons were requested to fill out a short questionnaire directly after each surgical case. The questionnaire included 3 questions to investigate surgeon satisfaction with the degree of mydriasis, with the degree of visualization due to mydriasis, and with stability of mydriasis. Surgeon satisfaction was rated on a scale of 1 (unsatisfied) to 5 (satisfied).

Patient-Reported Outcomes Postoperative pain was measured directly after the patient left the operating room using a visual analog scale on a scale of 0 to 10 . In addition, each patient was requested to fill out the Catquest-9SF questionnaire preoperatively (on the day of surgery) and 4 weeks postoperatively. The Catquest-9SF is a questionnaire with 9 items, designed to measure cataract-related visual disability and how it is affected by cataract surgery. Seven items assess specific tasks affected by visual functioning. The remaining 2 general items determine patient satisfaction with vision and overall vision-related difficulties in daily life. The Catquest-9SF is currently the predominant patient-reported outcome measure used in the evaluation of cataract care in the Netherlands. Each item is rated on a scale of 1 (no difficulties/very satisfied) to 4 (major difficulties/very unsatisfied). The sum scores of completed questionnaires (ie, no missing values) were converted to Rasch scores using a conversion table, which has previously been validated in a cohort of Dutch patients undergoing cataract surgery. Possible Rasch scores ranged from -6.14 (sum score 9, ie, best quality of vision) to 5.71 (sum score 36 , ie, worst quality of vision). ${ }^{12}$

\section{Statistical Analysis}

Sample Size Calculation The sample size calculation was performed separately for the comparison of ICMA vs the ocular insert and ICMA vs topical mydriatics and was based on a noninferiority design. It was estimated that the total costs of ICMA were $€ 4.13$ higher than that of the ocular insert and $€ 4.42$ higher than that of topical mydriatics. Assuming an SD in incremental costs of $€ 20$, a noninferiority margin of $€ 4.00$ (ie, the study is able to demonstrate a statistically significant difference in costs if the difference is at least $€ 4.00$ ), a power of 0.90 , and an $\alpha$ of 0.05 , the required sample size was 104 patients per group for the comparison of ICMA vs the ocular insert and 97 patients per group for the comparison of ICMA vs topical mydriatics.

Data Analysis Continuous variables were reported using means and SDs, whereas discrete variables were reported using frequency distributions. Statistical tests were applied to assess potentially statistically significant differences between groups. Because cost outcomes are typically highly skewed, it has been advocated to use nonparametric tests rather than parametric tests, with a special preference for the nonparametric bootstrap. ${ }^{13}$ In a nonparametric bootstrap, a sample of equal size is randomly drawn from the observed sample. Samples are drawn with replacement, meaning that the observed value of a single participant can be drawn multiple times. Subsequently, the statistic of interest (eg, mean) is calculated for the sample. This procedure is repeated multiple times, typically 1000 times, to arrive at a distribution for the statistic of interest, which can be used for further analysis. As such, a nonparametric bootstrap was applied using 1000 replications in Microsoft Excel 2016 for Windows (version 16.0; Microsoft Corp.). For every replication, the treatment with the lowest mean cost was selected, and the total number of replications in which each treatment was the least costly was determined.

Other outcome parameters were initially tested for overall differences between all 3 study groups using an $\mathrm{F}$ test (continuous variables) or a $\chi^{2}$ test (discrete variables). If the $P$ value was below the 0.05 threshold, additional statistical tests were performed to make direct group comparisons using a $t$ test (continuous variables) or a $\chi^{2}$ test. Testing was performed using IBM SPSS Statistics for Windows software (version 25.0; IBM Corp.).

\section{RESULTS}

A total of 368 patients were included in the study and allocated to topical mydriatics $(\mathrm{n}=131)$, the ocular insert $(\mathrm{n}=$ $115)$, and ICMA $(n=122)$. Baseline characteristics are summarized in Table 1 . There were no statistically significant differences in age and sex. There were also no statistically significant differences in the proportion of diabetic patients or patients using $\alpha$-blockers (both are believed to compromise mydriasis) or the cumulative dissipated energy during surgery, which is an indirect measure of the hardness and complexity of the cataract. In Table 2, the proportions of missing data are shown per study group for each outcome parameter. The proportion of missing data on Catquest-9SF Rasch scores was clearly higher than other outcome parameters. Although some questionnaires were completely missing ( $1.4 \%$ of all preoperative questionnaires and $4.3 \%$ of all postoperative questionnaires), most missing Rasch scores were missing because patients were able to fill in "cannot decide" as a possible answer to an item. In such case, the item was considered missing, and a Rasch score could no longer be determined using the described method.

\section{Cost Analysis}

Table 3 describes the mean resource use and mean costs per patient. Total mean costs were $€ 451$ (SD 134) in the topical mydriatics group, $€ 474$ (SD 129) in the ocular insert group, and $€ 506$ (SD 111) in the ICMA group. The acquisition cost of ICMA was higher than that of the ocular insert and topical mydriatics. Including the cost of the syringe and needle required to administer ICMA, the cost per patient was $€ 17$, compared with $€ 5$ for topical mydriatics and $€ 7$ for the ocular insert (includes the cost of the nurse administering the mydriatic). The number of patients requiring additional mydriasis intraoperatively 
Table 1. Baseline Characteristics.

\begin{tabular}{|c|c|c|c|c|}
\hline & Topical $(n=131)$ & Mydriasert $(n=115)$ & Mydrane $(n=122)$ & $P$ value \\
\hline Male sex, n (\%) & 57 (43.5) & $43(37.4)$ & $52(42.6)$ & .60 \\
\hline Age $(y)$, mean (SD) & $71.0(7.6)$ & $72.4(8.4)$ & $72.9(8.2)$ & .16 \\
\hline Diabetes, n (\%) & 27 (20.6) & $27(23.5)$ & $26(21.3)$ & .88 \\
\hline Use of $\alpha$-blockers, $n(\%)$ & $8(6.1)$ & $4(3.5)$ & $2(1.6)$ & .18 \\
\hline $\begin{array}{l}\text { Cumulative dissipated } \\
\text { energy, mean (SD) }\end{array}$ & $11.3(5.9)$ & $11.4(6.9)$ & $10.7(5.9)$ & .68 \\
\hline
\end{tabular}

${ }^{a} P$ value based on $\mathrm{F}$ test (continuous variables) or $\chi^{2}$ test (discrete variables)

was relatively evenly distributed (topical mydriatics group, 8 mydriatics in 8 patients [6.1\%]; ocular insert group, 6 mydriatics in 6 patients [5.2\%]; and ICMA group, 6 mydriatics in 5 patients [4.1\%]; $P=.521$ ). The more costly Malyugin ring was only used in the ocular insert and ICMA groups, 3 and 4 times, respectively (iris hooks were not used in any patients).

Differences in surgery time were a major contributor to total cost differences. Total surgery costs were $€ 388$ in the topical mydriatics group, $€ 411$ in the ocular insert group, and $€ 431$ in the ICMA group. The mean surgery time over all included patients was 14.13 minutes; however, Table 4 summarizes that there was a statistically significant variability in mean surgery times among surgeons, varying from 10.60 minutes for the fastest surgeon to 20.51 minutes for the slowest surgeon. Using a cutoff of 1.5 minutes within mean surgery time, all surgeons were categorized into fast surgeons $(<12.63$ minutes mean surgery time), average surgeons (12.63 to 15.63 minutes mean surgery time), or slow surgeons $(>15.63$ minutes mean surgery time). As shown in Figure 1, there was an imbalance in the distribution of surgeons over the 3 study groups, with nearly half $(44.3 \%, \mathrm{n}=58)$ of the topical mydriatics patients having been operated by fast surgeons, and only $16.8 \%(\mathrm{n}=22)$ by slow surgeons. In contrast, roughly half of the patients in the Mydriasert group (46.0\%, $\mathrm{n}=52)$ and Mydrane group $(51.2 \%, \mathrm{n}=62$ ) had been operated by slow surgeons. By contrast, roughly half of the patients in the ocular insert and ICMA groups had been operated by slow surgeons. The impact of other resources on total costs was negligible.

Bootstrap analysis revealed that, of 1000 replications, topical mydriatics was the least costly strategy in $90 \%$ of replications, the ocular insert in $10 \%$ of replications, and ICMA in $0 \%$ of replications. A subgroup analysis in which patients operated by the 2 fastest and slowest surgeons were excluded revealed that topical mydriatics was the least costly strategy in $64 \%$ of replications (mean costs $€ 444, \mathrm{n}=$ 71 ), the ocular insert in $36 \%$ of replications (mean costs $€ 450, \mathrm{n}=50$ ), and ICMA in $0 \%$ of replications (mean costs $€ 526, \mathrm{n}=57)$.

\section{Pupil Diameter}

The ratio of pupil size to white-to-white distance is reported in Table 5 and Figure 2. Although the mean pupil diameter remained relatively stable throughout the surgery in the topical mydriatics and the ocular insert groups, there was a clear increase in pupil diameter between $\mathrm{T} 1$ and $\mathrm{T} 3$ in the

Table 2. Percentage Missing Data Per Outcome Parameter.

\begin{tabular}{|c|c|c|c|}
\hline & Topical $(n=131)$ & Mydriasert $(n=115)$ & Mydrane $(n=122)$ \\
\hline \multicolumn{4}{|l|}{ Costs (\%) } \\
\hline Total healthcare costs & 5.3 & 5.2 & 0.8 \\
\hline Total patient and family costs & 6.1 & 7.0 & 2.5 \\
\hline Total costs & 6.1 & 8.7 & 3.3 \\
\hline \multicolumn{4}{|l|}{ Pupil diameter (\%) } \\
\hline T1, pupil size just before first corneal incision & 1.5 & 4.3 & 0.8 \\
\hline T2, pupil size just before injection of OVD & 0.8 & 2.6 & 0.8 \\
\hline T3, pupil size just before capsulorhexis & 0.8 & 2.6 & 0.8 \\
\hline T4 pupil size just before IOL implantation & 0.8 & 1.7 & 0 \\
\hline T5 pupil size just before end of surgery & 0.8 & 2.6 & 0 \\
\hline \multicolumn{4}{|l|}{ Surgeon-reported outcome measures (\%) } \\
\hline Satisfaction with degree of mydriasis & 4.6 & 5.2 & 1.6 \\
\hline Satisfaction with visualization due to mydriasis & 4.6 & 5.2 & 1.6 \\
\hline Satisfaction with stability of mydriasis & 4.6 & 5.2 & 1.6 \\
\hline \multicolumn{4}{|l|}{ Patient-reported outcomes (\%) } \\
\hline Preop Catquest-9SF Rasch score & 13.0 & 14.8 & 12.3 \\
\hline Postop Catquest-9SF Rasch score & 25.2 & 27.8 & 23.0 \\
\hline VAS pain score & 0 & 0 & 0 \\
\hline
\end{tabular}

OVD = ophthalmic viscosurgical device; postop = postoperative; preop = preoperative; $\mathrm{VAS}=$ visual analog scale 
Table 3. Mean Resource Use and Mean Costs (in 2019 €).

\begin{tabular}{|c|c|c|c|c|c|c|c|}
\hline & \multirow[b]{2}{*}{$\begin{array}{l}\text { Costs per } \\
\text { unit }(€)\end{array}$} & \multicolumn{3}{|c|}{ Resource use, mean (SD) } & \multicolumn{3}{|c|}{ Costs $(€)$, mean (SD) } \\
\hline & & $\begin{array}{l}\text { Topical } \\
(n=131)\end{array}$ & $\begin{array}{l}\text { Mydriasert } \\
(\mathrm{n}=115)\end{array}$ & $\begin{array}{l}\text { Mydrane } \\
(n=122)\end{array}$ & $\begin{array}{l}\text { Topical } \\
(n=131)\end{array}$ & $\begin{array}{l}\text { Mydriasert } \\
(\mathrm{n}=115)\end{array}$ & $\begin{array}{l}\text { Mydrane } \\
\text { ( } n=122)\end{array}$ \\
\hline \multicolumn{8}{|l|}{ Healthcare costs } \\
\hline \multicolumn{8}{|l|}{ Mydriatic } \\
\hline Topical & 2.80/unit & $1(-)$ & - & - & $3(-)$ & - & - \\
\hline Mydriasert & 6.31/unit & - & $1(-)$ & - & - & $6(-)$ & - \\
\hline Mydrane & 12.12/unit & - & - & $1(-)$ & - & - & $12(-)$ \\
\hline Additional materials (Mydrane only) & 4.60/unit & - & - & $1(-)$ & - & - & $5(-)$ \\
\hline Nurse time for administering mydriatic & $0.49 / \mathrm{min}$ & $4.5(0.2)$ & $1.6(0.4)$ & - & $2(0.1)$ & $1(0.2)$ & - \\
\hline \multicolumn{8}{|l|}{ Surgery costs } \\
\hline General operating room costs & $12.71 / \mathrm{min}$ & $26.4(7.6)$ & $28.1(6.8)$ & $29.5(5.8)$ & $336(96)$ & $358(87)$ & $375(74)$ \\
\hline Ophthalmology costs & $3.78 / \mathrm{min}$ & $13.6(6.0)$ & $14.1(5.4)$ & $14.7(5.1)$ & $52(23)$ & $53(20)$ & $56(19)$ \\
\hline \multicolumn{8}{|l|}{ Additional mydriatic } \\
\hline Intracameral phenylephrine 10\% & 1.15/unit & 0.05 (6 pt) & 0.01 (1 pt) & $0(0 \mathrm{pt})$ & $0(0.2)$ & $0(0.1)$ & $0(0)$ \\
\hline Topical & 1.65/unit & 0.02 (2 pt) & $0.02(2 \mathrm{pt})$ & 0.02 (2 pt) & $0(0.2)$ & $0(0.2)$ & $0(0.2)$ \\
\hline $\begin{array}{l}\text { Pupillary expansion device (Malyugin } \\
\text { ring) }\end{array}$ & 115.92/unit & 0 (0 pt) & 0.03 (3 pt) & 0.03 (4 pt) & $0(0)$ & $3(19)$ & $4(21)$ \\
\hline Postop nurse time relating to pain & $0.49 / \mathrm{min}$ & $0.3(2.0)$ & $0(0.1)$ & $0(0.5)$ & $0(1.0)$ & $0(0)$ & $0(0.2)$ \\
\hline Unplanned outpatient visits and calls & Variable & & Variable & & $1(11)$ & $0(0)$ & $2(11)$ \\
\hline Subtotal & & & & & $393(121)$ & 419 (115) & $454(103)$ \\
\hline \multicolumn{8}{|l|}{ Patient and family costs } \\
\hline Travel costs & Variable & & Variable & & $6(4)$ & $8(6)$ & $8(6)$ \\
\hline Postop analgesics & Variable & & Variable & & $0(0)$ & $0(0)$ & $0(0)$ \\
\hline Informal care & $14.65 / h$ & $3.5(2.9)$ & $3.2(2.8)$ & $3.3(3.3)$ & $51(42)$ & $47(41)$ & $48(48)$ \\
\hline Subtotal & & & & & $58(43)$ & $55(41)$ & 57 (49) \\
\hline Total costs & & & & & $451(134)$ & 474 (129) & 506 (111) \\
\hline
\end{tabular}

postop $=$ postoperative $; \mathrm{pt}=$ patients

ICMA group, after which it remained stable until the end of surgery. The mean ratio of pupil size to white-to-white distance was statistically significantly lower in the ICMA group compared with the topical mydriatics and the ocular insert groups at all timepoints $(P<.001$ at $\mathrm{T} 1$ to T5). There was no statistically significant difference between topical mydriatics and the ocular insert groups ( $P$ ranging from .11 to .82 ).

The pupil diameter was $6.0 \mathrm{~mm}$ or more at T3 (just before capsulorhexis) in $100 \%$ of patients in the topical

\begin{tabular}{|c|l|l|}
\hline \multicolumn{2}{|l|}{ Table 4. Surgery Times Per Surgeon. } \\
\hline & $\begin{array}{l}\text { Mean surgery } \\
\text { time (min) }\end{array}$ & No. of patients \\
\hline Fast surgeons $^{\mathrm{a}}$ & & \\
Surgeon 1 & 10.60 & 57 \\
Surgeon 2 & 11.15 & 26 \\
Surgeon 3 & 12.42 & 36 \\
Average surgeons & & \\
Surgeon 4 & 13.00 & 59 \\
Surgeon 5 & 13.13 & 30 \\
Surgeon 6 & 13.33 & 9 \\
Surgeon 7 & 13.92 & 12 \\
Slow surgeons & & \\
Surgeon 8 & 15.82 & 44 \\
Surgeon 9 & 15.96 & 47 \\
Surgeon 10 & 20.51 & 45 \\
\hline
\end{tabular}

$a_{<}<12.63$ minutes of mean surgery time

$b_{12.63}$ to 15.63 minutes of mean surgery time

$c_{>} 15.63$ minutes of mean surgery time mydriatics group, $97.3 \%$ of patients in the ocular insert group, and $90.9 \%$ of patients in the ICMA group, which was statistically significantly lower than the topical mydriatics $(P<.001)$ and the ocular insert $(P=.04)$ groups.

\section{Surgeon-Reported Outcomes}

Surgeon satisfaction with degree of mydriasis was highest in the topical mydriatics group with $96.8 \%$ of surgical cases

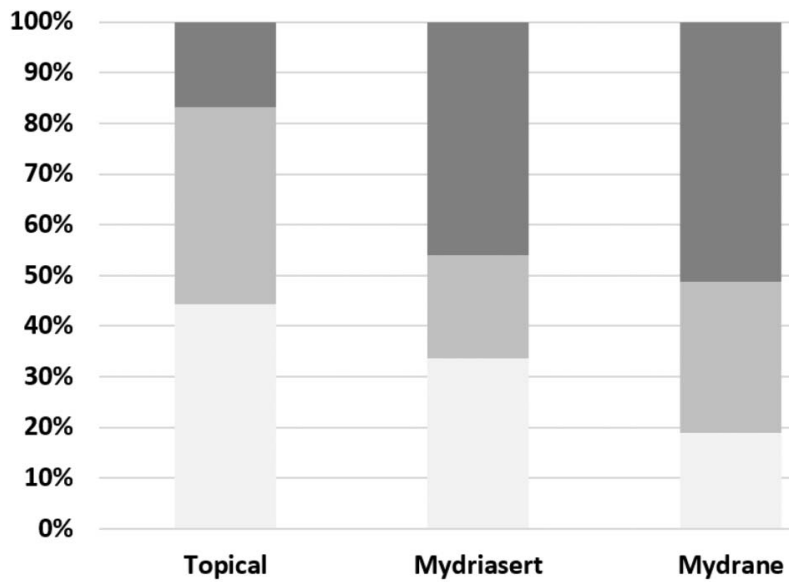

Fast surgeons $\llbracket$ Average surgeons $₫$ Slow surgeons

Figure 1. Proportion of fast surgeons $(<12.63$ minutes of mean surgery time), average surgeons (12.63 to 15.63 minutes of mean surgery time), and slow surgeons (>15.63 minutes of mean surgery time) for the topical mydriatics, Mydriasert, and Mydrane groups. 
Table 5. Pupil Size.

\begin{tabular}{|c|c|c|c|}
\hline & Topical $(n=131)$ & Mydriasert $(n=115)$ & Mydrane $(n=122)$ \\
\hline \multicolumn{4}{|c|}{ Ratio of pupil size to white-to-white distance, mean (SD) } \\
\hline T1 pupil size just before first corneal incision & $0.66(0.07)$ & $0.66(0.10)$ & $0.20(0.05)^{\mathrm{a}, \mathrm{b}}$ \\
\hline T2 pupil size just before injection OVD & $0.66(0.07)$ & $0.66(0.09)$ & $0.53(0.07)^{\mathrm{a}, \mathrm{b}}$ \\
\hline T3 pupil size just before capsulorhexis & $0.68(0.07)$ & $0.69(0.09)$ & $0.60(0.07)^{\mathrm{a}, \mathrm{b}}$ \\
\hline T4 pupil size just before IOL implantation & $0.68(0.07)$ & $0.67(0.09)$ & $0.62(0.08)^{\mathrm{a}, \mathrm{b}}$ \\
\hline T5 pupil size just before end of surgery & $0.67(0.07)$ & $0.66(0.08)$ & $0.62(0.08)^{\mathrm{a}, \mathrm{b}}$ \\
\hline \multicolumn{4}{|c|}{ Proportion eyes with pupil diameter $6 \mathrm{~mm}$ or more $(\%)^{\circ}$} \\
\hline T1 pupil size just before first corneal incision & 98.4 & 95.5 & $0^{e}, f$ \\
\hline T2 pupil size just before injection OVD & 99.2 & $94.6^{d}$ & $67.8^{\mathrm{e}, \mathrm{f}}$ \\
\hline T3 pupil size just before capsulorhexis & 100 & 97.3 & $90.9^{e}, f$ \\
\hline T4 pupil size just before $\mathrm{IOL}$ implantation & 100 & 97.3 & $91.8^{\mathrm{e}}$ \\
\hline T5 pupil size just before end of surgery & 100 & 97.3 & $92.6^{\circ}$ \\
\hline
\end{tabular}

OVD = ophthalmic viscosurgical device

a $P$ value $<.05$ for statistically significant difference between topical and Mydrane ( $t$ test)

${ }^{b} P$ value <.05 for statistically significant difference between Mydriasert and Mydrane ( $t$ test)

${ }^{c}$ Pupil diameter in millimeters calculated based on assumption that white-to-white distance in all eyes is $11.9 \mathrm{~mm}$

${ }^{\mathrm{d}} P$ value $<.05$ for statistically significant difference between topical and Mydriasert ( $\chi^{2}$ test)

${ }^{e} P$ value $<.05$ for statistically significant difference between topical and Mydrane ( $\chi^{2}$ test)

${ }^{f} P$ value $<.05$ for statistically significant difference between Mydriasert and Mydrane ( $\chi^{2}$ test)

rated 4 or higher (on a scale of 1 to 5), compared with $92.6 \%$ in the ocular insert group, and $76.6 \%$ in the ICMA group, which was statistically significantly lower than the topical mydriatics $(P<.001)$ and the ocular insert $(P=.001)$ groups (Figure 3$)$. There was no statistically significant difference between the topical mydriatics and ocular insert groups. Similar values were observed in surgeon satisfaction with visualization due to mydriasis, with $100 \%$ of surgical case rated 4 or higher in the topical mydriatics group, $93.6 \%$ in the ocular insert group [statistically significantly lower than the topical mydriatics group $(P=.004)]$, and $78.4 \%$ in the ICMA group [statistically significantly lower than the topical mydriatics $(P<.001)$ and ocular insert $(P=.001)$ groups] (Figure 4). Surgeon satisfaction with stability of mydriasis was more comparable between groups, with $99.2 \%$ of surgical cases rated 4 or higher in the topical mydriatics group, 93.6\% in the ocular insert group, which was statistically significantly lower than the topical mydriatics group $(P=.02)$, and $90.8 \%$

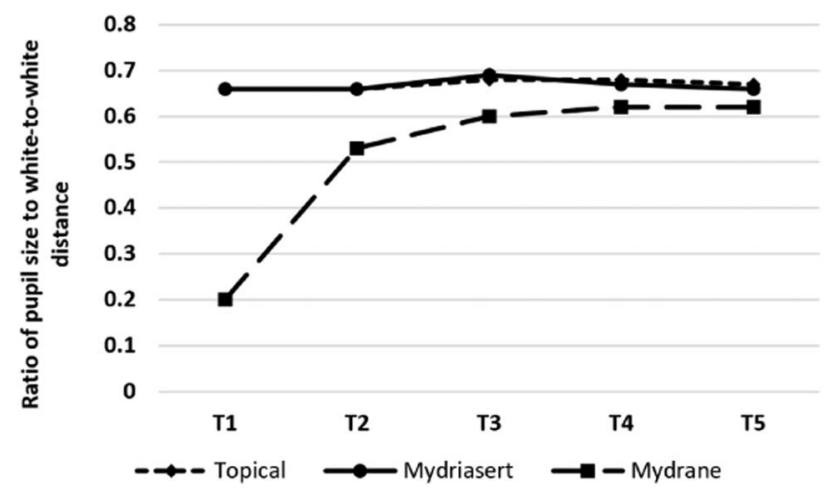

Figure 2. Ratio of pupil size to white-to-white distance during cataract surgery for the topical mydriatics, Mydriasert, and Mydrane groups. T1 = just before first corneal incision; T2 = just before injection of ophthalmic viscosurgical device; T3 = just before capsulorhexis; T4 = just before intraocular lens implantation; T5 = just before end of surgery. in the ICMA group, which was statistically significantly lower compared with the topical mydriatics group $(P=.002)$ but not with the ocular insert group (Figure 5).

\section{Patient-Reported Outcomes}

Patient-reported outcomes are reported in Table 6. The mean visual analog scale pain scores were less than 1 (on a scale of 1 to 10) in all study groups with no statistically significant differences between groups. Catquest-9SF Rasch scores showed a clear improvement after cataract surgery compared with baseline in all study groups. There were no statistically significant differences between groups in preoperative and postoperative Rasch scores.

\section{DISCUSSION}

This cost analysis compared 3 strategies to achieve mydriasis in cataract surgery in a Dutch healthcare setting. Topical mydriatics were the least costly strategy. The mean

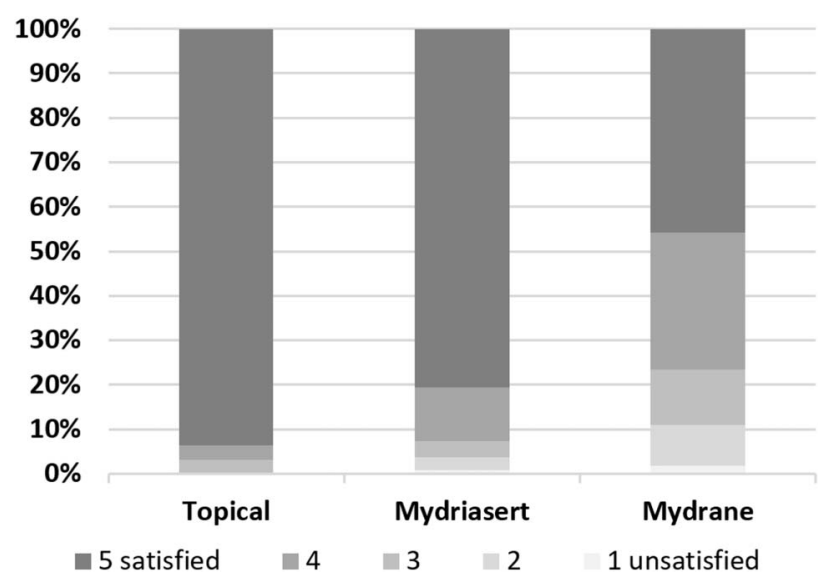

Figure 3. Surgeon-reported satisfaction with degree of mydriasis, rated on a scale of 1 (least satisfied) to 5 (most satisfied). 


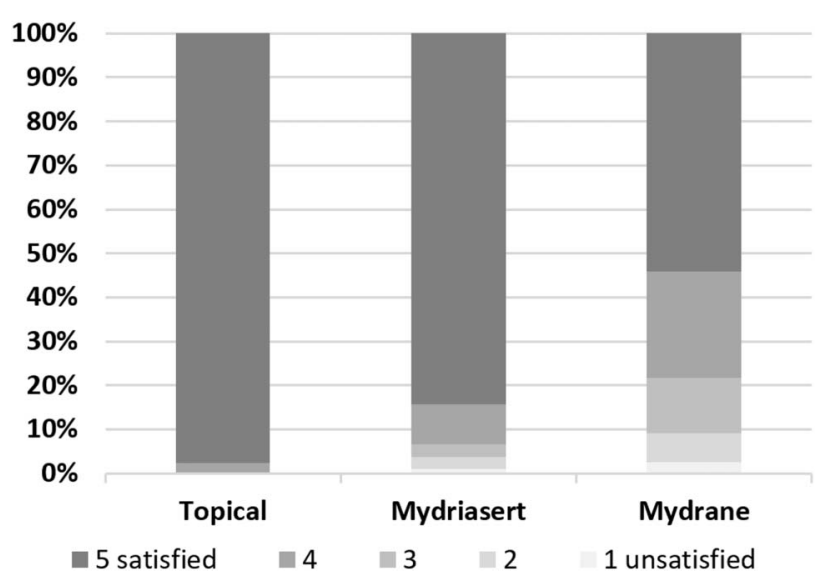

Figure 4. Surgeon-reported satisfaction with visualization due to mydriasis, rated on a scale of 1 (least satisfied) to 5 (most satisfied).

total costs per patient in the topical mydriatics group were $€ 23$ lower than that of the ocular insert group and $€ 55$ lower than that of the ICMA group. Important factors that contributed to the steeper costs in the ICMA group were the higher acquisition cost of the product and the additional materials needed to administer ICMA during surgery. Furthermore, use of ICMA was associated with an increase in surgical time, which further increased costs. Although ICMA did result in savings in nurse costs, the benefit was too small to outweigh the additional expenses. In this research setting, no adaptations were made in the perioperative process that would have taken advantage of the benefits of ICMA, such as a real-life setting where patients receiving ICMA would not need to arrive to the hospital until shortly before the planned surgery time.

An imbalance was found in the distribution of the proportion of patients having been operated by relatively slow and relatively fast surgeons over the 3 study groups, with more patients in the topical mydriatics group having been operated by fast surgeons and more patients in the ocular insert and ICMA groups having been operated by slow surgeons. This increased surgery costs in the latter 2 groups. Indeed, the $€ 23$ difference in total costs between the topical mydriatics and ocular insert groups could almost fully be explained by the observation that surgery costs were $€ 23$ higher in the ocular insert group. There was no statistically significant difference between these 2 groups in pupil size or the number of patients requiring additional mydriatics intraoperatively that could explain the difference in surgical costs.

It is important to note that the outcomes of this study may be different in other settings. For instance, costs of mydriatics, personnel, and surgery may differ between countries. Therefore, the results of this study may not directly be applicable in other countries. Transferability of the study results was enabled by reporting the volumes of resources use for most types of resources. The costs per unit reported in Table 3 can be substituted by local unit cost prices to calculate total costs.

The specific hospital setting may also influence the potential difference in costs. In this study, patients in all 3 study groups

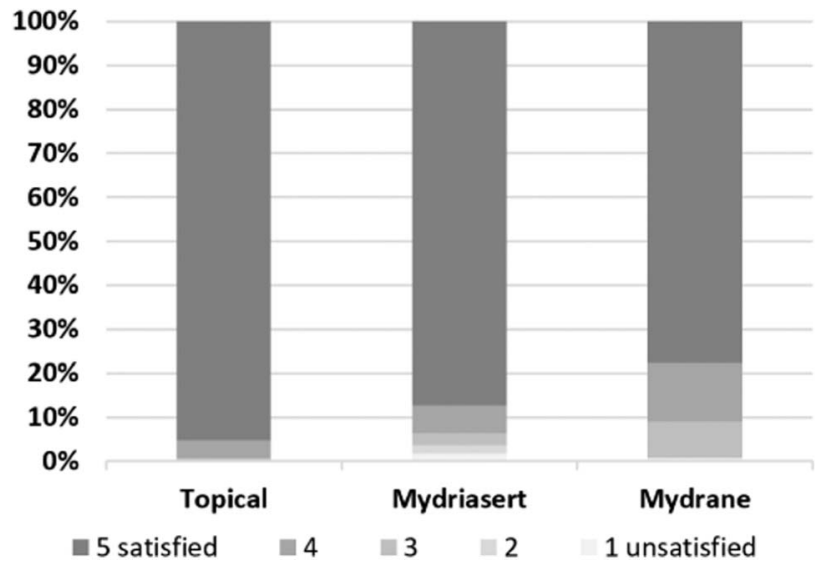

Figure 5. Surgeon-reported satisfaction with stability of mydriasis, rated on a scale of 1 (least satisfied) to 5 (most satisfied).

underwent the same perioperative procedures. The only difference was the method of mydriasis. However, it has been shown that implementation of ICMA dramatically reduces preoperative waiting times. ${ }^{5,14}$ This is especially relevant when limited waiting room capacity obstructs patient flow when using mydriasis techniques that warrant longer exposure times (topical mydriatics and ocular insert). Furthermore, contrary to the results of this study, a recent study showed that implementation of ICMA led to a reduction in surgical times and rotation times between patients.

It is conceivable that implementation of ICMA, depending on the real-life setting and local logistical characteristics, could be cost neutral or even cost saving when combined with structural alterations in the perioperative process, such as reducing the number of nurses or reducing the amount of physical space dedicated to perioperative patient care. Such alterations were not incorporated in the context of this study because of the significant logistical implications it would have.

As stated earlier, a previous U.K. budget impact model demonstrated that implementation of ICMA in a hospital setting resulted in slight cost savings compared with topical mydriatics. ${ }^{6}$ Although the acquisition costs of ICMA were higher than the acquisition costs of topical mydriatics, the costs were offset by savings in nurse costs. It should be noted that the difference in mydriatic acquisition costs in that study (ICMA $£ 6.00$; topical mydriatics $\mathfrak{E 1 . 1 1 )}$ ) was smaller than that in this current study (ICMA $€ 12.12$; topical mydriatics $€ 2.80$ ). In addition, the extra costs of a syringe and hydrodissection needle used to administer ICMA were not accounted for. Finally, in the model, it was assumed that nurses spent a total of 9 minutes on administering topical mydriatics. A sensitivity analysis revealed that ICMA was more costly than topical mydriatics when time for administering eyedrops was reduced to 4.5 minutes. A similar budget impact model has previously been conducted in the United Kingdom for ocular insert. ${ }^{15}$ Compared with topical mydriatics, ocular insert was associated with higher acquisition costs, which 
Table 6. Patient-Reported Outcomes.

\begin{tabular}{|l|l|l|l|l|}
\hline & Topical $(\mathbf{n}=\mathbf{1 3 1})$ & Mydriasert $(\mathbf{n}=\mathbf{1 1 5})$ & Mydrane $(\mathbf{n}=\mathbf{1 2 2})^{\boldsymbol{P} \text { value }} \mathbf{b}^{\mathrm{b}}$ & .65 \\
\hline VAS pain score (range 0 to 10), mean (SD) & $0.10(0.61)$ & $0.11(0.63)$ & $0.05(0.40)$ & .35 \\
Catquest-9SF preop Rasch score, mean (SD) & $-1.51(1.94)$ & $-1.66(1.72)$ & $-1.30(1.60)$ & .68 \\
Catquest-9SF postop Rasch score, mean (SD) & $-3.43(2.23)$ & $-3.68(2.11)$ & $-3.64(1.99)$ & \\
\hline
\end{tabular}

postop $=$ postoperative; preop $=$ preoperative; VAS $=$ visual analog scale

aLower score indicates better functioning (range -6.14 to +5.71 )

bP value based on $\mathrm{F}$ test

was offset by a reduction in nurse costs. As a result, ocular insert was a cost-saving strategy. However, in that study, nurses spent, on average, 1.5 minutes on administering ocular insert and 8.5 minutes on administering topical mydriatics, compared with 1.6 and 4.5 minutes, respectively, in this study.

In this study, the mean pupil diameter was lowest in the ICMA group and comparable in the ocular insert and topical mydriatics groups. This finding is in line with the results of previous studies, along with the finding that surgeon satisfaction with mydriasis was lower in the ICMA group. ${ }^{5,16-20}$ Despite the lower pupil diameter in the ICMA group, there was no statistically significant difference between groups in the number of patients who required additional intraoperative mydriatics. By contrast, a previous study found that $1.1 \%$ of patients receiving ICMA and $5.3 \%$ of patients receiving topical mydriatics required additional intraoperative mydriatics before successful capsulorhexis could be achieved. ${ }^{5}$ In addition, mydriasis remained more stable throughout the surgery after administration of ICMA compared with topical mydriatics. ${ }^{16}$

Strengths of this study include its relatively large sample size, the inclusion of out-of-hospital costs in the cost analysis to provide a more comprehensive impression of the economic impact of ICMA, and the real-life hospital setting in which the study was conducted, increasing external validity. However, a few weaknesses should be addressed. First, the study was nonrandomized. Although there were no changes in the perioperative process in the course of the study that could act as confounders, bias could not be ruled out. For instance, some surgeons predominantly operated patients in 1 or 2 study groups. Because not all surgeons operated equally fast, with more patients having been operated by relatively slow surgeons in the ICMA and ocular insert groups, this led to a bias in surgical costs. Second, patient inclusion in each study group was started immediately after the method for achieving mydriasis was changed to another mydriatic. Surgeons and nurses were not able to get familiar with the new mydriatic. However, the learning curve for using the ocular insert and ICMA is presumably relatively short. Third, the study was conducted in a single hospital, and differences in logistical procedures between hospitals may impact the results of the study. Fourth, pupil diameter was not measured in millimeters but in number of pixels using stills of video recordings. Although this method may theoretically be more accurate than measurements performed intraoperatively with calipers, it is more difficult to compare the results with other studies and to determine the proportion of patients with a pupil diameter above a certain cutoff value. Pupil size to white-to-white distance ratios were converted to pupil diameter in millimeters using a mean reported white-to-white distance of $11.9 \mathrm{~mm}$, but this will certainly have led to overestimations and underestimations in individual patients. ${ }^{5} \mathrm{Fi}$ nally, surgeons were asked to rate their satisfaction with visualization due to mydriasis. However, the method used to achieve mydriasis might affect visualization because of not only the degree of mydriasis but also alterations in ocular surface integrity. The latter was not enquired.

To conclude, based on this cost analysis performed in a single hospital in the Netherlands, ICMA was more costly when implemented as a substitute for topical mydriatics or ocular insert in cataract surgery. In addition, ICMA was associated with smaller, albeit stable, pupil size. Nonetheless, implementation of ICMA may be of value when part of a larger operational redesign program to make cataract surgery and the perioperative process leaner, especially in the setting of high-volume, low-complexity cataract surgery clinics. The ocular insert, which is more comparable with topical mydriatics in costs and pupil size, could also be considered a suitable alternative.

\section{WHAT WAS KNOWN}

- The intracameral mydriatics and anesthetic agent (ICMA) is a safe and efficient method to achieve rapid and stable mydriasis during cataract surgery.

- The mydriatic ocular insert is a safe method to achieve mydriasis prior to cataract surgery, similar to topical mydriatics.

- Both the ICMA and mydriatic ocular insert reduce preoperative workload for nurses, and ICMA additionally reduces time spent by patients in the surgical center prior to cataract surgery.

\section{WHAT THIS PAPER ADDS}

- In a Dutch healthcare setting, both the ICMA and the mydriatic ocular insert were associated with a higher acquisition cost than topical mydriatics, which were not offset by cost savings resulting from a lower nurse workload.

- Although intraoperative pupil size was similar in patients who received topical mydriatics or the mydriatic ocular insert, pupil size was significantly smaller in patients who received the ICMA. Surgeon satisfaction with mydriasis was correspondingly lower in surgical cases in which ICMA was administered. 


\section{REFERENCES}

1. Lundström M, Goh P, Henry Y, Salowi MA, Barry P, Manning S, Rosen P, Steveni $U$. The changing pattern of cataract surgery indications: a 5-year study of 2 cataract surgery databases. Ophthalmology 2015;122:31-38

2. Rijksinstituut voor Volksgezondheid en Milieu. Zorguitgaven staar naar sector. Available at: https://www.volksgezondheidenzorg.info/onderwerp/ gezichtsstoornissen/kosten/zorguitgaven\#node-zorguitgaven-staar-naarsector. Accessed March 19, 2020

3. Guell J, Behndig A, Pleyer U, Jaulerry S, Rozot P, Pisella P, Robert P, Lanzl I, Pourjavan S, Aguiar C, Fernandez J, Grabner G, Mencucci R, Chiambaretta F, Labetoulle M. Systemic exposure to intracameral vs topical mydriatic agents: in cataract surgery. Clin Ophthalmol 2019;13:811-819

4. Behndig A, Korobelnik J. Mydriatic insert and intracameral injections compared with mydriatic eyedrops in cataract surgery: controlled studies. J Cataract Refract Surg 2015;41:1503-1519

5. Labetoulle M, Findl O, Malecaze F, Alió J, Cochener B, Lobo C, Lazreg S, Hartani D, Colin J, Tassignon M, Behndig A, Intracameral Mydrane Study 2 Group. Evaluation of the efficacy and safety of a standardised intracameral combination of mydriatics and anaesthetics for cataract surgery. $\mathrm{Br} \mathrm{J}$ Ophthalmol 2016;100:976-985

6. Davey K, Chang B, Purslow C, Clay E, Vataire A. Budget impact model of Mydrane, a new intracameral injectable used for intra-operative mydriasis, from a UK hospital perspective. BMC Ophthalmol 2018;18:104

7. Hadj Salah WB, Rousseau A, M'garrech M, Best AL, Barreau E, Labetoulle M. Assessment of the duration of surgery and patient turnover after the incorporation of a standardized intracameral combination of mydriatics and anesthetics for cataract surgery. J Cataract Refract Surg 2020;46: 1487-1494

8. Hakkaart-van Roijen L, van der Linden N, Bouwmans CA, Kanters T, Tan SS. Kostenhandleiding: methodologie van kostenonderzoek en referentieprijzen voor economische evaluaties in de gezondheidszorg. Diemen, the Netherlands: Zorginstituut Nederland; 2015

9. Centraal Bureau voor de Statistiek. Consumer prices; price index 2015=100. Available at: https://opendata.cbs.nl/statline/\#/CBS/en/dataset/83131eng/table?ts=1584630934048. Accessed March 19, 2020

10. Zorginstituut Nederland. Medicijnkosten. Available at: https://www.medicijnkosten.nl. Accessed March 20, 2020

11. Merkala. Available at: https://www.merkala.nl. Accessed March 20, 2020

12. Visser MS, Dieleman M, Klijn S, Timman R, Lundström M, Busschbach JJ, Reus NJ. Validation, test-retest reliability and norm scores for the Dutch Catquest-9SF. Acta Ophthalmol 2017;95:312-319

13. Desgagné A, Castilloux AM, Angers JF, LeLorier J. The use of the bootstrap statistical method for the pharmacoeconomic cost analysis of skewed data. Pharmacoeconomics 1998;13(5 pt 1):487-497
14. Schulz CB, Goverdhan SV, Humphry RC. An evaluation of intracameral mydriasis for routine cataract surgery. $\mathrm{Br} \mathrm{J}$ Ophthalmol 2018;102: 784-789

15. Shah A, Johal S, Lee N. Mydriasert pupillary dilation for cataract surgery: an economic and clinical study. BMC Ophthalmol 2015;15:56

16. Chiambaretta F, Pleyer U, Behndig A, Pisella PJ, Mertens E, Limao A, Fasce F, Fernandez J, Benmoussa SE, Labetoulle M, Cochener B, Intracameral Mydrane (ICM) and Ethics Group. Pupil dilation dynamics with an intracameral fixed combination of mydriatics and anesthetic during cataract surgery. J Cataract Refract Surg 2018;44:341-347

17. Morgado G, Barros P, Martins J, Lima A, Martins N. Comparative study of mydriasis in cataract surgery: topical versus Mydriasert versus intracameral mydriasis in cataract surgery. Eur J Ophthalmol 2010;20:989-993

18. de Faria A, Giorgi R, Cohen Salmon M, Bonnel S, Holweck G, Le Corre A Chazalon E. Same-eye comparison of pupillary dilation with an intraoperative standardized intracameral combination of mydriatics (Mydrane) versus a preoperative ophthalmic (Mydriasert) in standard cataract surgery in nondiabetic patients. J Fr Ophtalmol 2019;42:e339-e348

19. Saenz-de-Viteri M, Fernández-Robredo $P$, de Nova $E$, Bonet-Farriol E, Sabater AL, Zarranz-Ventura J, Caire J, Sádaba LM, Salinas-Alamán A, García-Layana A. Comparative study measuring the dilatory effect of a mydriatic device (Mydriasert) versus topical drops. Int J Ophthalmol 2013;6: 801-804

20. Torrón C, Calvo P, Ruiz-Moreno O, Leciñena J, Pérez-Iñigo A. Use of a new ocular insert versus conventional mydriasis in cataract surgery. Biomed Res Int 2013;2013:849349

Disclosures: R.M.M.A. Nuilts is a consultant at Alcon Laboratories, Inc., and Laboratoires Théa, receives financial support for research from Alcon Laboratories, Inc., Bausch \& Lomb, Inc., and Ophtec, and receives lecture fees from Alcon Laboratories, Inc., and Laboratoires Théa. N. Visser receives lecture fees from Novartis. R.M.P.C. de Crom is a consultant at Allergan, Inc., and Laboratoires Théa and receives lecture fees from Iridex and Novartis. All other authors report no financial disclosures.

\section{First author: \\ Rob W.P. Simons, MD}

University Eye Clinic Maastricht, Maastricht

University Medical Center, Maastricht, the Netherlands 